\title{
Monitor de Frequência Respiratória Utilizando Dois Acelerômetros
}

\author{
Clóvis Garcia Marcondes ${ }^{1}$, Leonardo Vidal Batista ${ }^{1}$, Alisson V. de Brito ${ }^{1}$ \\ ${ }^{1}$ Centro de Informática - Universidade Federal da Paraíba (UFPB) \\ João Pessoa - PB - Brazil \\ clovisgarciadgmail.com, \{leonardo,alisson\}@ci.ufpb.br
}

\begin{abstract}
The respiratory rate $(R R)$ is an important information source about an individual health condition however, the options available, in addition to be very expensive, require a controlled environment and/or the usage of equipment not suitable for routine use. For data acquisition for measurement of $R R$, is proposed the use of two accelerometers, being one frontal to allow the reading of acceleration forces exerted on the trunk by the action of respiration and another on the back used as reference of movement perception. The objective of this work is to demonstrate the feasibility of such apparatus, allowing its use in vital sign monitoring projects.
\end{abstract}

Resumo. A frequência respiratória (FR) é um elemento que pode oferecer informações importantes sobre a condição do individuo, contudo as opções disponiveis no mercado, além de terem alto custo, requerem um ambiente controlado elou o uso de equipamentos que certamente não seriam adequados para o uso rotineiro. Para a aquisição de dados para a aferição da FR, sugerese a utilização de dois acelerômetros, sendo um frontal com o objetivo de capturar as forças exercidas sobre o tronco do indivíduo pelas ações de inspiração e expiração e um posterior utilizado como referência para percepção de movimento. O objetivo deste trabalho é demonstrar a viabilidade de tal aparato, permitindo seu uso em projetos de monitoramento de sinais vitais.

\section{Introdução}

O monitoramento dos sinais vitais é um elemento importante, tanto no acompanhamento de pacientes hospitalizados, como aqueles que estão fora do ambiente hospitalar mas requerem cuidados específicos. Outra área onde tal atividade é extremamente relevante é na prática de atividades físicas, tanto amadoras quanto profissionais. Em particular, no caso da FR, a falta de equipamentos eficientes, não invasivos e que permitam um acompanhamento em tempo real das informações acabam por prejudicar o bem estar das pessoas, e até mesmo comprometer ações que poderiam evitar o agravamento de doenças ou situações atípicas reveladas por seus sinais vitais. Mesmo no caso do acompanhamento de pacientes hospitalizados que apresentam problemas respiratórios, existem estudos que demonstram a ineficiência no registro da FR (Cretikos et al., 2008).

Em parte isso se deve à dificuldade em se realizar a correta verificação desse sinal de forma eficiente, apesar de sua relevância. Dispositivos que possam monitorar a respiração, normalmente são de 3 tipos distintos: os que medem o fluxo de ar, aqueles que avaliam composição ou movimento dos tecidos do abdômen ou tórax e os que avaliam a concentração gasosa no sangue ou ar expelido (Bates et al., 2010). Dentre os 
métodos propostos, o uso de acelerômetros surgiu recentemente como uma alternativa não invasiva para tal medição. Bates também indica a ausência de maiores estudos prévios considerando os impactos da movimentação do indivíduo sobre os valores medidos pelos acelerômetros, o que tornaria inviável o seu uso.

A prática de atividade física tem se intensificado nos últimos anos. A cada dia vê-se um aumento no número de pessoas que procuram alguma atividade física, seja com o propósito de manutenção de saúde, hobby ou com objetivos de sociabilização. Alguns acabam por se engajar de uma forma mais intensa, porém ainda a nível amador. Em particular, tem-se visto um aumento dos adeptos de corridas de rua, que por sua simplicidade no que diz respeito à prática da atividade em si, a qual requer poucos equipamentos, acaba por ser uma escolha mais confortável. Além da prévia avaliação médica, em especial de um cardiologista, o acompanhamento de profissionais de educação física é algo bastante importante, tanto para a correta avaliação das capacidades do atleta, seja pela orientação da atividade em si. Não é incomum observar praticantes de corridas efetuarem eventualmente exames especificamente montados para avaliar suas capacidades físicas, de forma a orientar o treinamento a ser realizado. Em especial, a realização de exames, como a ergoespirometria, fornecem informações importantes sobre como o organismo do indivíduo se encontra.

O Limiar Anaeróbico (LA) é uma dessas informações, a qual evidencia o ponto a partir do qual o metabolismo aeróbico perde sua eficiência e o anaeróbico passa a ser mais exigido. A partir do LA, a produção de ácido lático alcança valores superiores ao que o organismo consegue reutilizar. Com isso começa a ocorrer acúmulo do mesmo, o qual acaba por desencadear o processo de fadiga muscular, prejudicando a performance do atleta e, a depender da manutenção dessa situação, fazendo com que o mesmo tenha que interromper a atividade. Uma das formas de se controlar a realização das atividades físicas, observando-se o LA, é através do monitoramento cardíaco. Os monitores cardíacos têm se popularizado, como um meio de acompanhar e, de certa forma, corretamente dosar a intensidade da atividade física dentro de uma faixa adequada às condições fisiológicas do indivíduo.

A informação cardíaca, apesar de ser extremamente relevante, nem sempre é suficiente para dar um correto feedback ao praticante de atividade física sobre as suas condições fisiológicas frente à atividade física. A depender da duração da atividade física, fatores como o nível de hidratação e temperatura corporal acabam por exigir uma readequação da frequência cardíaca, de forma compensatória. Com isso, mesmo mantendo o mesmo nível de atividade física, acaba-se por observar um aumento da frequência cardíaca, o qual naturalmente faz com que o indivíduo reduza seu ritmo de atividade de forma precipitada. Além do mais, com o passar do tempo, com o treinamento, o indivíduo adquire uma melhor condição física, mas tendo em vista as dificuldades para a realização de novos exames com uma menor frequência, acabam por não ajustar o seu ritmo de treino ou, em algumas situações, sem o devido embasamento fazem ajustes dos treinos sem considerar se de fato já conseguem atingir aquele nível.

Outros sinais vitais possuem relevância, tais como pressão arterial, temperatura, FR, sudorese, além também das condições onde a atividade está sendo realizada, como por exemplo, temperatura ambiente, umidade, altitude, etc. Pela falta de equipamentos acessíveis, cômodos e práticos para a coleta e processamento desses sinais, acabamos muitas vezes por não realizar as atividades de forma segura e eficiente. Tanto podemos 
estar realizando a mesma abaixo da capacidade do nosso corpo, e com isso não tendo um desenvolvimento adequado, como também podemos estar extrapolando as condições fisiológicas ou negligenciando sinais que o corpo esteja produzindo de algo não está bem, e uma ação deve ser tomada.

Vê-se portanto, que a medição da FR é um importante indicador, tanto com relação à saúde do indivíduo, como para no caso de práticas esportivas, para melhor eficiência, por sua correlação com o LA (Carey et al., 2005). O presente trabalho propõe um mecanismo prático e de baixo custo, para a medição da FR, mesmo na ocorrência de movimento dos indivíduos. Ele se baseia na utilização de acelerômetros para essa medição. Esses sensores, além do fato de possuírem baixo custo e tamanho reduzido, são capazes de avaliar deformações mínimas, como aquelas apresentadas no tronco durante o ciclo de respiração, representados por mudanças periódicas na angulação dos acelerômetros (Hung et al., 2008). Isso abre espaço para o desenvolvimento de aplicativos assistentes, com o objetivo de processar essas informações e utilizá-las na identificação de padrões anormais ocorridos durante a realização das atividades.

\section{Materiais e Métodos}

\subsection{Protótipo}

Para montagem do protótipo, optou-se pela utilização da plataforma ARDUINO, tendo em vista sua flexibilidade e custo. O equipamento utilizado foi o Arduino UNO R3, que trata-se de uma placa baseada no microcontrolador ATmega 328, contendo 14 pinos de E/S digitais e 6 analógicos. A possibilidade de se trabalhar com componentes $I^{2} \mathrm{C}$ é uma grande vantagem dessa plataforma, permitindo a incorporação de diversos itens simultaneamente, sem comprometer a utilização de muitos pinos. Apenas precisamos ter uma preocupação com a corrente requerida, tendo em vista as limitações existentes tanto da placa de forma geral (200mA), como de cada pino de forma independente (40mA). Como atualmente dispomos de sensores com baixíssimo consumo e corrente, isso acaba não sendo um grande problema.

Foram escolhidos acelerômetros tri-axiais do tipo Micro Electro-Mechanical System (MEMS), dado seu reduzido tamanho e peso (Figueiredo et al., 2007), sendo que a utilização de uma placa breakout - placa de circuito para facilitar conexão dos pinos de um componente eletrônico - para os acelerômetros foi adotada pela simplicidade das conexões, e optou-se pelo modelo MMA8452Q da SparkFun (Figura 1), utilizando o acelerômetro de mesmo nome da Freescale, dadas suas características técnicas, disponibilidade e custo. Esse acelerômetro já possui um filtro Passa Alta incorporado, que pode ser ativado em tempo real, dois pinos de interrupção configuráveis, voltagem compatível com Arduino e escala de medição variável $(2 \mathrm{~g} / 4 \mathrm{~g} / 8 \mathrm{~g})$. Em nosso caso, tendo em vista que a aceleração da respiração é bem discreta e se pretende fazer as avaliações em situações de movimentação humana, usamos a opção de maior sensibilidade (2g), aumentando a precisão.

A informação da FR é enviada através da porta serial do equipamento, podendo ser capturada por uma aplicação externa, a qual pode apresentar visualmente tal informação, ou mesmo utilizá-la em conjunto com outras informações para a realização de algum processamento. 


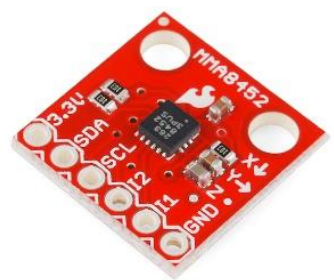

Figura 1- Placa breakout com acelerômetro MMA8452Q da SparkFun

\subsection{Metodologia Experimental}

Após a montagem do protótipo (Figura 2), foram realizados testes preliminares a fim de identificar a melhor configuração dos acelerômetros. O componente utilizado permite operação nas seguintes frequências, com respectivo consumo de corrente entre parênteses: $1.56 \mathrm{~Hz}(6 \mu \mathrm{A}), 6.25 \mathrm{~Hz}(6 \mu \mathrm{A}), 12.5 \mathrm{~Hz}(6 \mu \mathrm{A}), 50 \mathrm{~Hz}(14 \mu \mathrm{A}), 100 \mathrm{~Hz}(24 \mu \mathrm{A})$, $200 \mathrm{~Hz}(44 \mu \mathrm{A}), 400 \mathrm{~Hz}(85 \mu \mathrm{A})$ e $800 \mathrm{~Hz}(165 \mu \mathrm{A})$. Nesses testes observou-se que, mesmo a frequência de $1.56 \mathrm{~Hz}$ sendo capaz de capturar informações referentes à respiração, a curva dos processos de inspiração e expiração não possuía dados suficientes para ser formada, o que dificultaria o trabalho de processamento dos sinais. Como o consumo de energia das frequências de $1.56 \mathrm{~Hz}, 6.25 \mathrm{~Hz}$ e $12.5 \mathrm{~Hz}$ são iguais, optou-se por este último por apresentar um conjunto maior de dados a serem analisados, sem comprometimento do consumo de energia. Explorou-se as frequências mais altas para observar os dados que seriam coletados e, além de não agregarem ganhos significativos na precisão das informações, eles acabavam por sofrer alta interferência de ruídos devido à alta frequência. Sendo assim, a frequência de $12.5 \mathrm{~Hz}$ foi escolhida para ser utilizada nos experimentos deste trabalho.

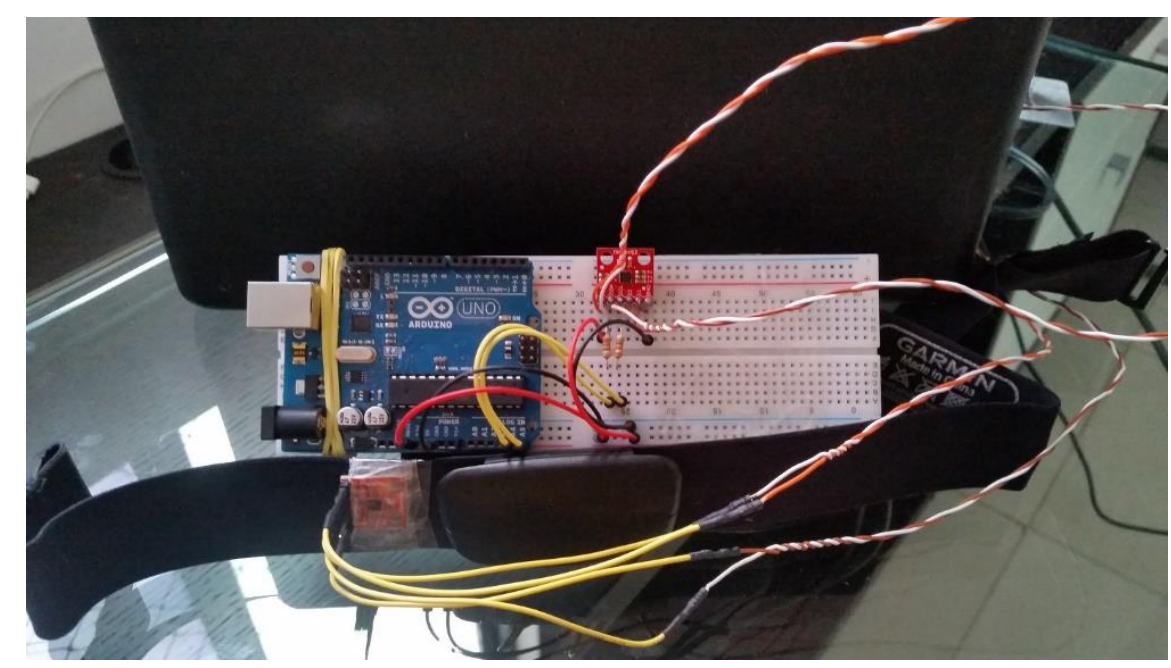

Figura 2- Protótipo utilizado nos experimentos

Com base nessa amostragem foram realizados quatro diferentes testes, todos contendo 10 respirações, sendo 3 em repouso e 1 delas caminhando: 
- Respiração normal (Figura 4)

- Respiração profunda (Figura 5)

- Respiração curta (Figura 6)

- Respiração normal, caminhando (Figura 7)

Importante ressaltar, que apesar dos acelerômetros utilizados possuírem nativamente a possibilidade de utilização de filtro de Passa Alta em tempo real, o mesmo não foi ativado. Isso se deve ao fato de que a principal vantagem no uso desse filtro, em se tratando desses acelerômetros, é a remoção das forças gravitacionais, permitindo que os 3 eixos, estando o acelerômetro em repouso, retornem valores próximos a 0 (ZERO). Como a aplicação irá fazer a subtração das resultantes dos dois acelerômetros, isso já será naturalmente eliminado, e portanto não há benefício no uso do mesmo.

Por outro lado, aplicou-se um filtro passa baixa nos valores retornados em cada eixo, de forma a reduzir um pouco do ruído presente no sinal. Foi utilizado o algoritmo Exponential Moving Average (EMA), com coeficiente $\alpha=0.1$ para estes experimentos. Tal algoritmo faz com que apenas parte (representado por $\alpha$ ) do dado observado em um dado momento seja efetivamente considerado, eliminando com isso parte do ruído.

Para efeitos comparativos, as figuras com os resultados apresentam os dados íntegros representados em AZUL, e os dados resultantes dos vetores que tiverem o filtro de Passa Baixa aplicado representados em AMARELO. Os dados apresentados dizem respeito ao resultado final, contendo a diferença $\left(\bar{R}=R_{\text {frontal }}-R_{\text {traseiro }}\right)$ das resultantes $(R=$ $\left.\sqrt{x^{2}+y^{2}+z^{2}}\right)$ de cada acelerômetro.

\section{Desenvolvimento}

A FR diz respeito à taxa de ventilação, ou seja, o número de respirações (ciclo de inspiração-expiração) medidos dentro de uma determinada faixa de tempo (tipicamente 60 segundos). O processo de respiração, que envolve o preenchimento e posterior esvaziamento dos pulmões com ar, provoca alterações nas estruturas torácicas e abdominais, em decorrência das alterações de volume de seus órgãos (Figura 3). Partindo da premissa de que tais estruturas não são sólidas, e apresentam em sua constituição mecanismos que permitem a distensão e, posterior, relaxamento à posição original, podemos considerar que tal movimentação pode ser medida através da aceleração à qual a superfície do corpo é submetida. Por este motivo, o uso do acelerômetro pode ser uma solução viável para alcançar o objetivo deste trabalho. 


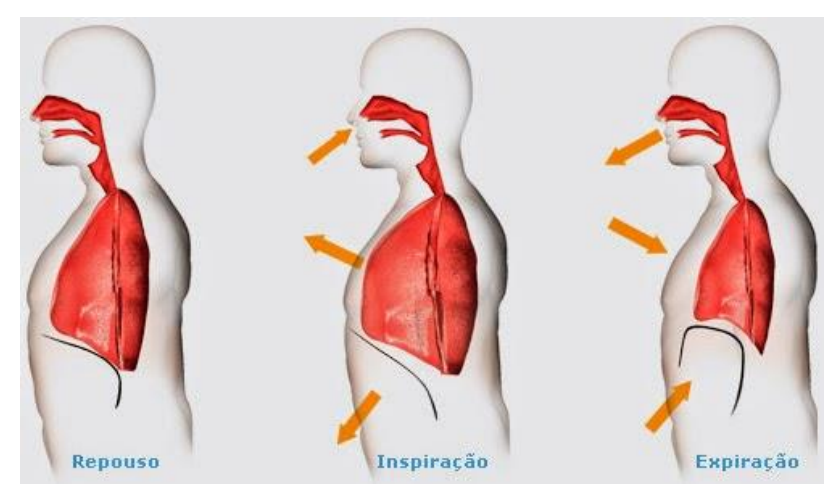

Figura 3 - Efeitos da Respiração no Corpo

Observando-se a anatomia do corpo humano e a dinâmica da alteração estrutural durante a respiração, observa-se que a linha medial, entre o tórax e o abdômen, na região precordial (região localizada imediatamente à frente do coração, à esquerda da porção inferior do esterno), apresenta o local ideal para medição, pois tanto as deformações torácicas, quanto as abdominais seriam possíveis de serem avaliadas, ao mesmo tempo que sofre menos interferência de rotação do tronco durante a movimentação. Outra razão para escolher tal posição é que também se refere à posição onde geralmente se utilizam as cintas dos frequencímetros, que os praticantes de atividade física já estão acostumados. Esta cinta pode ser utilizada para base de fixação dos sensores, evitando desconforto de mais um equipamento ou acessório preso ao comporto do indivíduo. Nota-se que, apesar de mais pronunciadas na direção frontal, as deformações anatômicas provocadas pela expansão de tórax e abdômen também ocorrem lateralmente, sendo portanto possível a utilização do acelerômetro em qualquer dessas posições. Contudo, a colocação frontal apresenta-se mais interessante, devido aos valores previstos para a leitura, tendo em vista se tratar de uma movimentação discreta, serem baixos. Essa maior margem de trabalho permite utilizar, com mais segurança, filtragem dos dados recebidos, eliminando ruídos oriundos de outras alterações e/ou movimentações do sensor.

Soluções baseadas em acelerômetro como a de Bates et al. (2010) e a de Hung et al. (2008) têm sido propostas, com o objetivo de utilização basicamente em ambientes hospitalares. Nessas condições, temos os pacientes geralmente em repouso em leitos. Contudo, mesmo nestes casos, algoritmos mais elaborados precisaram ser desenvolvidos de forma a se eliminar o efeito de movimentações do paciente, para não comprometer a medição. Nessas condições, a contagem das respirações não pode ser feita de forma eficiente pois não é possível se distinguir com facilidade a aceleração provocada pela movimentação do corpo como um todo, da movimentação provocada pela respiração. Como o objetivo seria a construção de um equipamento capaz de ser utilizado durante atividades físicas, onde obviamente precisar-se-ia ter o indivíduo em movimento, uma abordagem diferente precisou ser implementada. Optou-se pela utilização de um segundo acelerômetro, sendo este fixado às costas, logo atrás da coluna vertebral, o qual não sofreria influência das forças referentes à respiração mas, assim como ocorre com o primeiro acelerômetro, estaria sujeito às forças relativas à movimentação do corpo. Desta forma, tem-se uma base de referência do movimento e assim elimina-se o efeito do primeiro acelerômetro, tendo como resultado apenas os efeitos provocados pela respiração. Em um trabalho recente, Yoon et al. (2014) sugere uma abordagem similar para contornar os problemas advindos da movimentação, mas ao invés de utilizar um 
segundo acelerômetro eles optam por um giroscópio. Em um trabalho futuro, com os dados devidamente filtrados, poderemos comparar os nossos resultados com os deles.

A utilização do segundo acelerômetro também auxilia no processo de remoção da resultante da gravidade a qual, salvo quando o corpo se encontra em queda livre, apresenta uma aceleração contínua, em direção contrária ao centro da Terra. Como não é possível garantir o correto alinhamento dos sensores, pois os mesmos estarão em contato com o corpo e não seria prático estabelecer com precisão o devido alinhamento (seria necessário fazer uso de uma estrutura rígida, além de uma calibragem no início de toda medição), trabalha-se com a resultante dos diversos eixos. Por esse motivo, não existe nenhuma precondição para o alinhamento dos eixos dos sensores, mas a utilização de sensores triaxiais se faz necessário, de forma a podermos ter uma correta leitura das forças existentes nas 3 dimensões.

\section{Resultados}

Apesar de terem sido executados diversos testes para cada uma das situações previstas para este trabalho, os gráficos abaixo apresentados se referem a dados de um único experimento da situação indicada. É possível, visualmente, identificar os momentos de respiração, principalmente nos dados que passaram pelo filtro de Passa Baixa, contudo o reconhecimento dos padrões de respiração em termos computacionais ficará para um próximo trabalho.

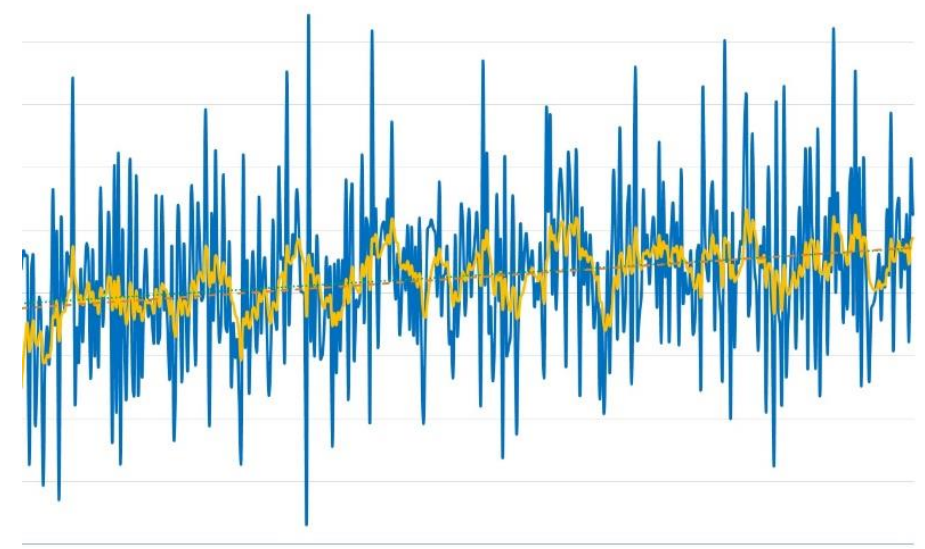

Figura 4 - Respiração Normal

Durante a respiração profunda, onde apesar da maior expansão das paredes torácica e abdominal, temos um movimento mais lento, e por conseguinte uma menor aceleração. Nesse caso, observa-se um maior espaçamento entre os picos, com uma menor altura relativa dos mesmos, devido à baixa aceleração. Dessa forma, temos uma maior influência dos ruídos na sobreposição aos dados da respiração. 


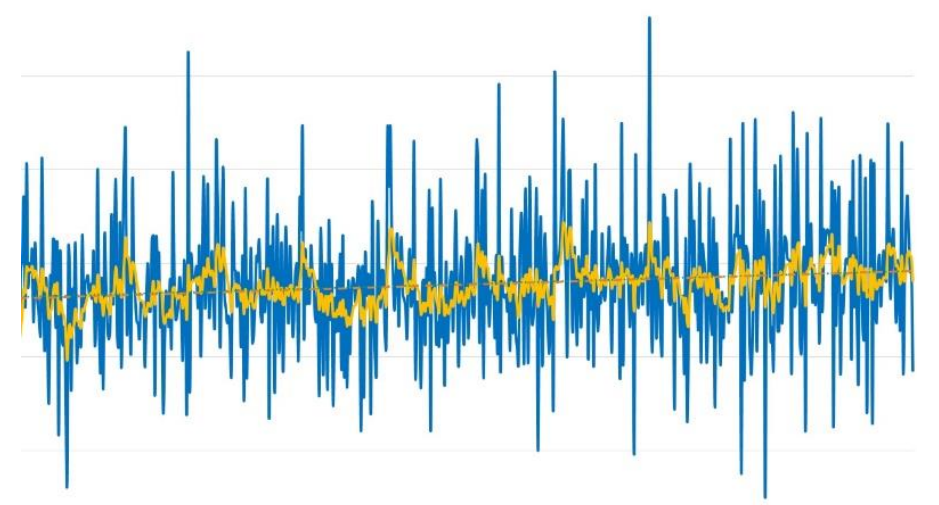

Figura 5 - Respiração Profunda

A respiração curta é a que apresenta o gráfico mais "limpo", tendo em vista que as acelerações são mais acentuadas de forma a podermos aumentar o processo respiratório.

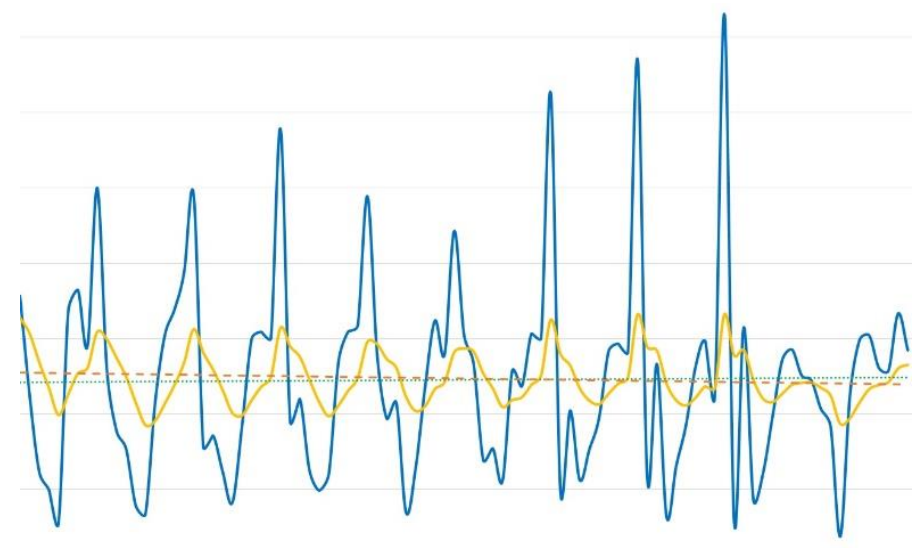

Figura 6 - Respiração Curta (acelerada)

Durante a caminhada observa-se uma perturbação muito maior nos dados retornados, motivados tanto pelo movimento em si, que faz com que as resultantes retornem valores maiores e, portanto as oscilações também acabem sendo maiores, como também por ruídos extras causados pela precariedade do protótipo, o qual apresentação muita oscilação.

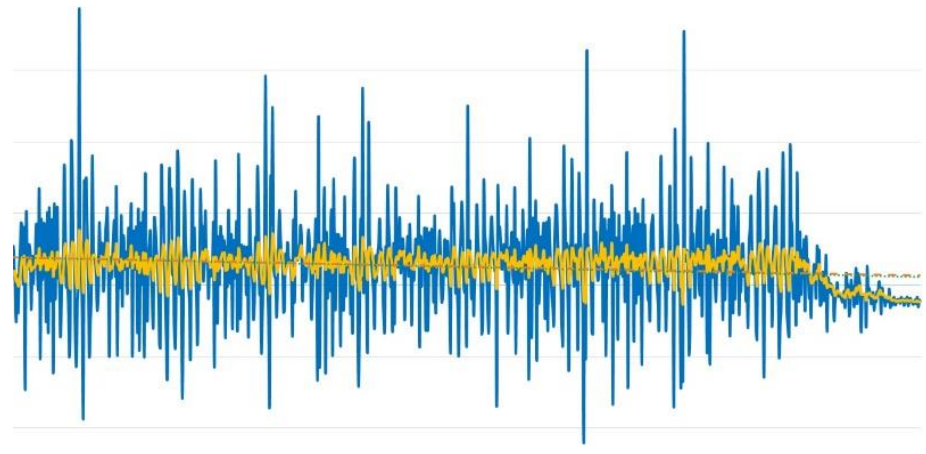

Figura 7 - Respiração Normal durante uma Caminhada 


\section{Conclusão}

A proposta de utilização de dois acelerômetros para a medição da FR se mostrou viável, apesar de ainda requerer um melhor processamento dos dados obtidos, bem como um refinamento na escolha da família de acelerômetros para a solução final, tendo em vista aspectos de custo, consumo e sensibilidade. A possibilidade de utilização de um equipamento com as características apresentadas neste trabalho, abre espaço para uso tanto esportivo como também na parte de prevenção de situações de saúde.

Em trabalhos futuros, trabalhar-se-á com maior intensidade no processamento dos sinais, a fim de eliminar mais ruídos, permitindo maior confiabilidade em situações mais extremas, como corridas. A sugestão do uso do filtro de Kalman (Figueiredo et al., 2007) será avaliada. Uma vez que se tenha sucesso na remoção do ruído e se obtenha uma onda mais suave representando a respiração, se poderá trabalhar na análise de picos para computar a FR. Deve-se levar em consideração, que no cômputo da resultante, como utiliza-se o módulo das componentes de aceleração, tanto a inspiração quanto a expiração apresentarão variação na aceleração, e a FR será contabilizada justamente na ocorrência de um evento de cada tipo.

Com relação aos experimentos, uma melhoria no protótipo a fim de que o mesmo possa armazenar as informações coletadas, para posterior análise está sendo considerada, para que mais informações possam ser capturadas. Também deve-se melhorar a qualidade da fixação dos acelerômetros para que nos testes em maior velocidade (corrida), tenha-se menos interferências pela movimentação do conjunto como um todo. Existe a possibilidade de que o acréscimo na velocidade de deslocamento degrade a qualidade dos dados de tal forma que torne inviável a separação dos dados de respiração, e portanto novos trabalhos poderão determinar limites de utilização da solução, ou necessidades de novos mecanismos de filtragem para cada patamar de velocidade.

Para o trabalho de processamento dos sinais, tanto se poderá optar por um sistema embarcado independente, como o uso de um smartphone (Bardolet and Glowacz, 2014), consolidando uma Wireless Body Sensor Network (WBSN), através da qual tanto se poderá incorporar novos sensores, como também montar uma plataforma ubíqua e pervasiva para o monitoramento de sinais vitais dos indivíduos.

\section{References}

Carey, D. G., Schwarz, L. A., Pliego, G. J. and Raymond, R. L. "Respiratory Rate Is a Valid and Reliable Marker for the Anaerobic Threshold: Implications for Measuring Change in Fitness", Journal of Sports Science and Medicine 4, pp. 482-488, 2005.

Cretikos, M. A., Bellomo, R., Hillman, K., Chen, J., Finfer, S. and Flabouris, A. "Repiratory rate: the neglected vital sign", $M J A$, vol. 188, number 11, pp. 657-659, 2008.

Bates, A., Ling, M. J., Mann J. and Arvind D. K. "Respiratory rate and flow waveform estimation from tri-axial accelerometer data", International Conference on Body Sensor Networks, 2010.

Figueiredo, L. J., Gafaniz, A. R., Lopes, G. S. and Pereira, R. "Aplicações de Acelerômetros", IAS 2007 - Instrumentação e Aquisição de Sinais. Lisboa, 2007. 
Hung, P., Bonnet, S., Guillemand, R., Castelli, E. and Yen, P. "Estimation of respiratory waveform using an accelerometer", Biomedical Imaging: From Nano to Macro, 2008. ISBI 2008. 5th IEEE International Symposium, pp. 1493-1496, 2008.

Yoon, J., Noh, Y., Kwon, Y., Kim, W. and Yoon, H. "Improvement of Dynamic Respiration Monitoring Through Sensor Fusion of Accelerometer and Gyro-sensor", Journal of Electrical Engineering \& Technology, Vol. 9, No. 1, pp. 334-343, 2014.

Bardolet, A. C. and Glowacz, A. "A Remote Patient Monitoring System using Android Mobile Devices”. Akademia Górniczo-Hutnicza Im. Stanislawa Staszica. 30th June 2014.

"Respiratory Rate." Wikipedia: The Free Encyclopedia. Wikimedia Foundation, Inc.. 20nd January 2015. Web. 29nd January 2015. $<$ http://www.wikiwand.com/en/Respiratory_rate>.

"MMA8452Q." Freescale Semiconductor. Data Sheet: Technical Data. 01st February 2015.

$<\mathrm{http}: / /$ www.freescale.com/webapp/sps/site/prod_summary.jsp?code=MMA8452Q $>$. 